\title{
Performance of the Medical Research Council (MRC) and the Leukemia Research Foundation (LRF) score in predicting survival benefit with hypomethylating agent use in patients with relapsed or refractory acute myeloid leukemia
}

Maximilian Stahl, Michelle DeVeaux, Pau Montesinos, Raphaël Itzykson, Ellen K. Ritchie, Mikkael A. Sekeres, John Barnard, Nikolai A. Podoltsev, Andrew Brunner, Rami S. Komrokji, Vijaya R. Bhatt, Aref Al-Kali, Thomas Cluzeau, Valeria Santini, Gail J. Roboz, Pierre Fenaux, Mark Litzow, Amir T. Fathi, Sarah Perreault, Tae Kon Kim, Thomas Prebet, Norbert Vey, Vivek Verma, Ulrich Germing, Juan Bergua, Josefina Serrano, Steven D. Gore \& Amer M. Zeidan

To cite this article: Maximilian Stahl, Michelle DeVeaux, Pau Montesinos, Raphaël Itzykson, Ellen K. Ritchie, Mikkael A. Sekeres, John Barnard, Nikolai A. Podoltsev, Andrew Brunner, Rami S. Komrokji, Vijaya R. Bhatt, Aref Al-Kali, Thomas Cluzeau, Valeria Santini, Gail J. Roboz, Pierre Fenaux, Mark Litzow, Amir T. Fathi, Sarah Perreault, Tae Kon Kim, Thomas Prebet, Norbert Vey, Vivek Verma, Ulrich Germing, Juan Bergua, Josefina Serrano, Steven D. Gore \& Amer M. Zeidan (2018): Performance of the Medical Research Council (MRC) and the Leukemia Research Foundation (LRF) score in predicting survival benefit with hypomethylating agent use in patients with relapsed or refractory acute myeloid leukemia, Leukemia \& Lymphoma, DOI: 10.1080/10428194.2018.1468893

To link to this article: https://doi.org/10.1080/10428194.2018.1468893

View supplementary material $[\pi$ 


\title{
Performance of the Medical Research Council (MRC) and the Leukemia Research Foundation (LRF) score in predicting survival benefit with hypomethylating agent use in patients with relapsed or refractory acute myeloid leukemia
}

\author{
Maximilian Stahl ${ }^{\mathrm{a}}$, Michelle DeVeaux ${ }^{\mathrm{b}}$, Pau Montesinos ${ }^{c}$, Raphaël Itzykson ${ }^{\mathrm{d}}$, Ellen K. Ritchie ${ }^{\mathrm{e}}$ \\ Mikkael A. Sekeres ${ }^{f}$, John Barnard ${ }^{f}$, Nikolai A. Podoltsev ${ }^{\mathrm{a}}$, Andrew Brunner ${ }^{\mathrm{g}}$, Rami S. Komrokji ${ }^{\mathrm{h}}$, \\ Vijaya R. Bhatt', Aref Al-Kali ${ }^{i}$ (D), Thomas Cluzeauk, Valeria Santini', Gail J. Roboz ${ }^{\mathrm{e}}$, Pierre Fenaux ${ }^{\mathrm{d}}$, \\ Mark Litzow $^{j}$ (D), Amir T. Fathi ${ }^{9}$, Sarah Perreault ${ }^{m}$, Tae Kon Kim ${ }^{a}$, Thomas Prebet ${ }^{a}$ (D), Norbert Vey ${ }^{n}$, \\ Vivek Vermai, Ulrich Germing ${ }^{\circ}$, Juan Bergua ${ }^{p}$, Josefina Serrano ${ }^{q}$, Steven D. Gore ${ }^{a}$ and Amer M. Zeidan ${ }^{a}$

\begin{abstract}
${ }^{a}$ Department of Internal Medicine, Section of Hematology, Yale University School of Medicine, New Haven, CT, USA; ${ }^{b}$ Department of Biostatistics, Yale School of Public Health, New Haven, CT, USA; 'Department of Medicine, University of Valencia, Hospital Universitario y Politécnico La Fe, Valencia, CIBERONC, Instituto III, Madrid, Spain; ${ }^{d}$ Department of Hematology/Oncology, Saint-Louis

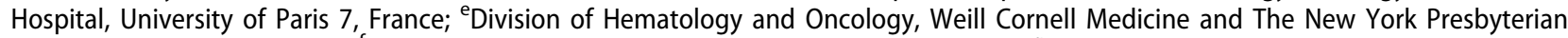
Hospital, New York, NY, USA; 'Leukemia Program Cleveland Clinic, Cleveland, OH, USA; ${ }^{9}$ Massachusetts General Hospital Cancer Center Harvard Medical School, Boston, MA, USA; ${ }^{h}$ Department of Malignant Hematology, Moffitt Cancer Center and Research Institute, Tampa, FL, USA; 'Division of Hematology/Oncology, University of Nebraska Medical Center, Omaha, NE, USA; ${ }^{\mathrm{j}} \mathrm{Mayo}$ Clinic, Rochester, MN, USA; ${ }^{k}$ Cote d'Azur University, Nice Sophia Antipolis University, CHU of Nice, Nice, France; 'Division of Hematology, University of Florence, Florence, Italy; ${ }^{\mathrm{m}}$ Department of Pharmacy, Yale New Haven Hospital, New Haven, CT, USA; ${ }^{\mathrm{n} D e p a r t m e n t}$ of Hematology, Institut Paoli Calmettes, Marseille, France; ${ }^{\circ}$ Department of Hematology, Oncology and Clinical Immunology, HeinrichHeine-University Duesseldorf, Duesseldorf, Germany; ${ }^{\mathrm{p}}$ Hospital San Pedro Alcántara, Cáceres, Spain; ${ }^{\mathrm{q}}$ Division of Hematology/
\end{abstract} \\ Oncology, University Hospital Reina Sofia, Cordoba, Spain
}

ARTICLE HISTORY Received 30 January 2018; revised 18 March 2018; accepted 16 April 2018

Patients with primary refractory or relapsed-acute myeloid leukemia (RR-AML), particularly older adults, have dismal outcomes and limited therapy options [1]. Given the tolerability of hypomethylating agents (HMAs) and ability to administer therapy on an outpatient basis, as well as limitations to clinical trial availability and eligibility, some patients with RR-AML are treated with the HMAs azacitidine or decitabine [1,2]. Using a large, international, multicenter database of RR-AML patients treated with HMAs, we have previously reported that $16 \%$ of patients achieved a complete response (CR) or CR with incomplete count recovery (CRi) with $\mathrm{HMAs}$ and experienced a median overall survival (OS) of 21 months [3].

However, similar to the situation in the frontline therapy setting, responses occur in a minority of patients and identification of clinical or laboratory predictors, which might help select patients who are likely to benefit from HMA therapy is ideal [4]. The Medical Research Council (MRC) and Leukemia Research Foundation (LRF) developed a score, recently published in Leukemia and Lymphoma [5], which predicts that older AML patients benefit from azacitidine as frontline therapy. However, little is known how this risk assessment tool performs in the refractory and relapsed setting, or among patients treated with decitabine. Here we analyzed the performance of the MRC/LRF tool in the prediction of OS in RRAML patients after therapy with HMAs using our large, international, multicenter database.

Our database included retrospectively collected data from 655 patients with RR-AML who were treated with HMAs in the 2006 to 2016 period from seven centers in the United States and five centers in Europe. Kaplan-Meier methods estimated OS and one-year OS from initiation of HMAs to death or end of follow-up. Patients who underwent allogeneic hematopoietic cell transplantation (alloHCT) were censored at the time of transplantation. Survival analysis was stratified according to the MRC/LRF risk categories of good, standard, and poor risk. Covariates for the MRC/LRF score include age, Eastern Cooperative Oncology Group Performance Status (ECOG PS), white blood cell count (WBC) at HMA initiation, AML type (de novo or secondary) and cytogenetic risk category according to MRC (favorable, intermediate, adverse). Missing data were imputed using the Multivariate Imputation by Chained Equation approach [6]. There were 10 imputed datasets generated from a

CONTACT Amer M. Zeidan $\otimes$ amer.zeidan@yale.edu @ Department of Internal Medicine, Section of Hematology, Yale University School of Medicine, Yale New Haven Health System, New Haven, CT 06519-1304, USA

The results of this research were presented in a poster presentation in the American Society of Hematology, Atlanta, GA, December 2017.

(C) 2018 Informa UK Limited, trading as Taylor \& Francis Group 
Table 1. Patient characteristics.

\begin{tabular}{|c|c|c|}
\hline Patient characteristic & Median or $N$ & Range or \% \\
\hline Age, years $(n=636)$ & 65 & $16-92$ \\
\hline \multicolumn{3}{|l|}{ Gender $(n=655)$} \\
\hline Male & 381 & 58.2 \\
\hline Female & 274 & 41.8 \\
\hline \multicolumn{3}{|l|}{ AML type $(n=650)$} \\
\hline De novo & 458 & 70.5 \\
\hline Secondary & 192 & 29.5 \\
\hline \multicolumn{3}{|l|}{ Disease status $(n=655)$} \\
\hline Relapsed AML & 365 & 55.7 \\
\hline Primary treatment refractory $\mathrm{AML}$ & 290 & 44.3 \\
\hline ECOG Performance Status $(n=137) 0$ & 50 & 36.5 \\
\hline 1 & 67 & 48.9 \\
\hline 2 & 18 & 13.1 \\
\hline 3 & 2 & 1.5 \\
\hline \multicolumn{3}{|l|}{ MRC cytogenetic risk group prior to initiation of HMA $(n=225)$} \\
\hline Good & 4 & 1.8 \\
\hline Intermediate & 131 & 58.2 \\
\hline Poor & 90 & 40 \\
\hline \multicolumn{3}{|l|}{ Chromosomal abnormalities } \\
\hline Complex karyotype $(n=224)$ & 54 & 24.1 \\
\hline Monosomy karyotype $(n=234)$ & 37 & 15.8 \\
\hline Chromosome 7 abnormalities $(n=224)$ & 50 & 22.3 \\
\hline Chromosome 5 abnormalities ( $n=224)$ & 44 & 19.6 \\
\hline Number of therapy lines prior to HMA $(n=648)$ & 1 & $1-7$ \\
\hline alloSCT prior to initiation of HMA (months) $(n=618)$ & 118 & 19.1 \\
\hline Duration of CR1 (months) prior to initiation of HMA for patients who achieved a CR1 $(n=322)$ & 8.0 & $0.5-180$ \\
\hline \multicolumn{3}{|l|}{ HMA agent used $(n=634)$} \\
\hline Azacitidine & 360 & 56.8 \\
\hline Decitabine & 274 & 43.2 \\
\hline
\end{tabular}

model with 68 variables using random forest imputation with 500 trees for missing continuous and unordered categorical data, and polytomous logistic regression for missing ordered categorical data. These methods restricted the imputed data to take plausible values found within the original data.

Of the 655 patients, 365 patients (56\%) had relapsed AML and 290 (44\%) had primary refractory AML (Table 1). Median age at start of HMA therapy was 65 years (range [R], 16-92) and the median ECOG PS was 1. Median WBC at HMA therapy was $3.2 \times 10^{9} / \mathrm{L}\left(\mathrm{R}, 0.1-110.5 \times 10^{9} / \mathrm{L}\right)$ and $22.4 \%, 4.4 \%$, and $0.2 \%$ of patients had a WBC of $>10 \times 10^{9} / \mathrm{L},>50 \times 10^{9} / \mathrm{L}$, and $>100 \times 10^{9} / \mathrm{L}$, respectively. Although 458 patients (70\%) had de novo AML, 192 patients had secondary AML (30\%). Only $1.8 \%$ of patients demonstrated a good risk karyotype based on MRC cytogenetic risk, whereas $58.2 \%$ and $40 \%$ of patients had an intermediate or poor risk karyotype.

Applying the MRC/LRF score, $20.9 \%$ of patients were categorized as a good risk, and hence more likely to benefit from therapy HMA, whereas $24.7 \%$ and $54.3 \%$ of patients were categorized as standard and poor risk, respectively. While 360 patients $(56.8 \%)$ were treated with azacitidine, 274 patients received decitabine (43.2\%). The median OS for the entire group of patients was 6.7 months (95\% Cl, 6.1-7.3). As expected, the median OS for responding patients was longer than that of nonresponding patients within each risk category of the MRC/LRF score. Median OS for patients treated with either azacitidine or decitabine (Figure 1) was 6.8 months for patients with either good or standard risk and 5.8 months for patients with poor risk based on MRC/LRF (hazard ratio [HR] of death for the poor risk group compared with the good/standard risk group was 1.3, $p=.090)$. The one-year OS probability was $29.2 \%(95 \% \mathrm{Cl} 17.8-41.2 \%)$ versus $19.6 \%(95 \% \mathrm{Cl}$ 9.6-32.0\%) for good/standard risk and poor risk patients, respectively $(p=.13)$ (Figure 1). For the subgroup of patients treated with azacitidine, median OS for patients with good/standard versus poor risk was 8.5 months compared with 6.2 months (HR $=1.2, p=.23$ ) (Figure 2(A)). The one-year OS probability was $32.7 \%$ (95\% Cl 24.2-41.4\%) versus 24.2\% (95\% Cl 18.1-30.7\%) for poor versus good/standard $(p=.059)$. For patients treated with decitabine, the difference in OS based on MRC/LRF was less pronounced with good/standard risk compared with poor risk patients experiencing a median OS of 5.6 and 5.3 months, respectively ( $H R=1.2, p=.28$ ). The one-year OS probability was $25.7 \% \quad(95 \% \quad \mathrm{Cl}$ $15.9-36.7 \%)$ vs. $15.4 \%$ (95\% Cl 9.0-23.3\%), respectively $(p=.33)$ (Figure 2(B)).

There was no significant difference in OS based on the type (azacitidine vs. decitabine) and schedule (5 vs. 7 vs. 10 day cycle) of HMA received, as well as disease status (primary refractory vs. relapsed), number of treatment lines ( 1 vs. 2 vs. 3 or more) or the transplant status (HSCT received vs. not) prior to HMA therapy.

The cause of death was reported for 292 patients (55\%) of the 529 patients who died during the follow-up. The most common cause of death was progression of disease/relapse (63.0\%), whereas other causes of death 

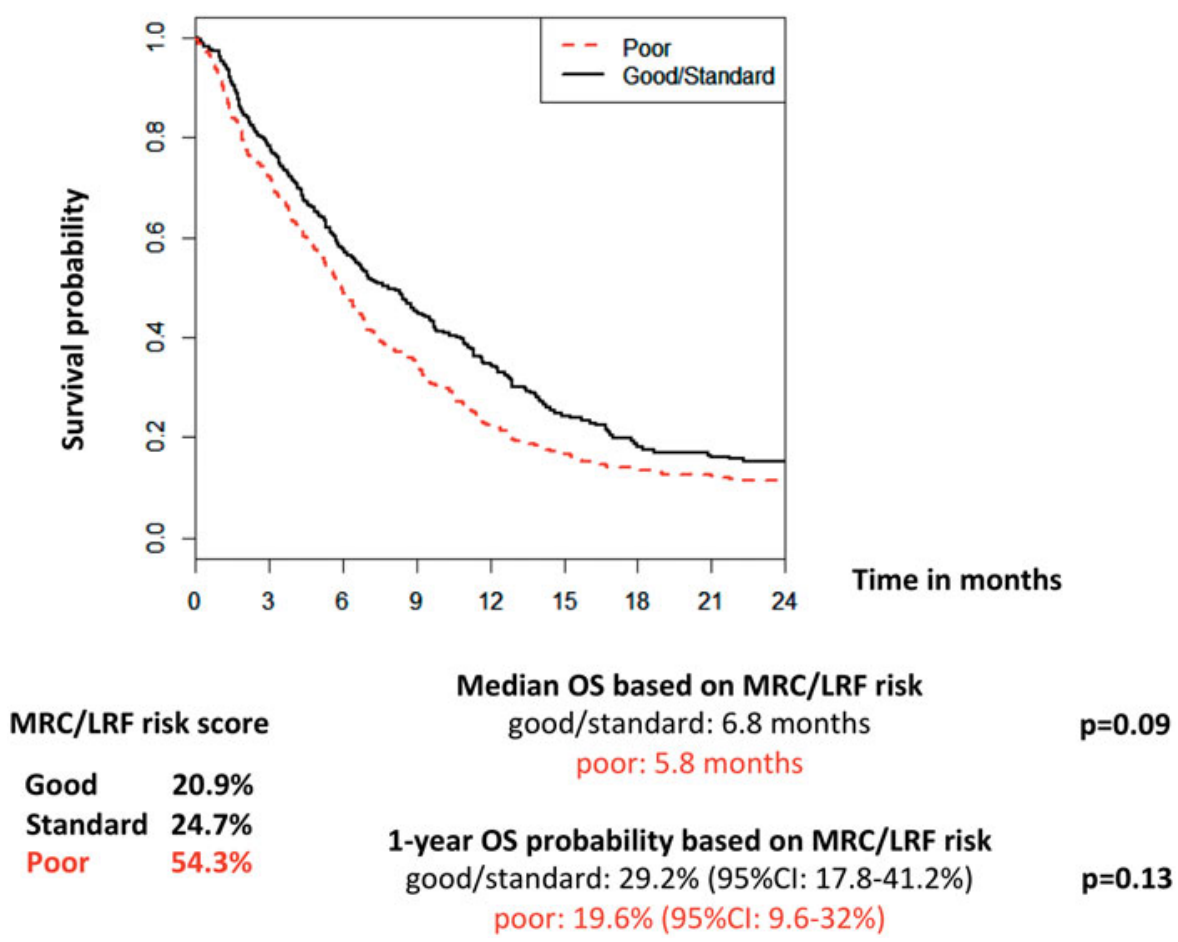

Figure 1. Probability of overall survival from onset of HMA treatment according to MRC/LRF risk category (good/standard vs. poor).

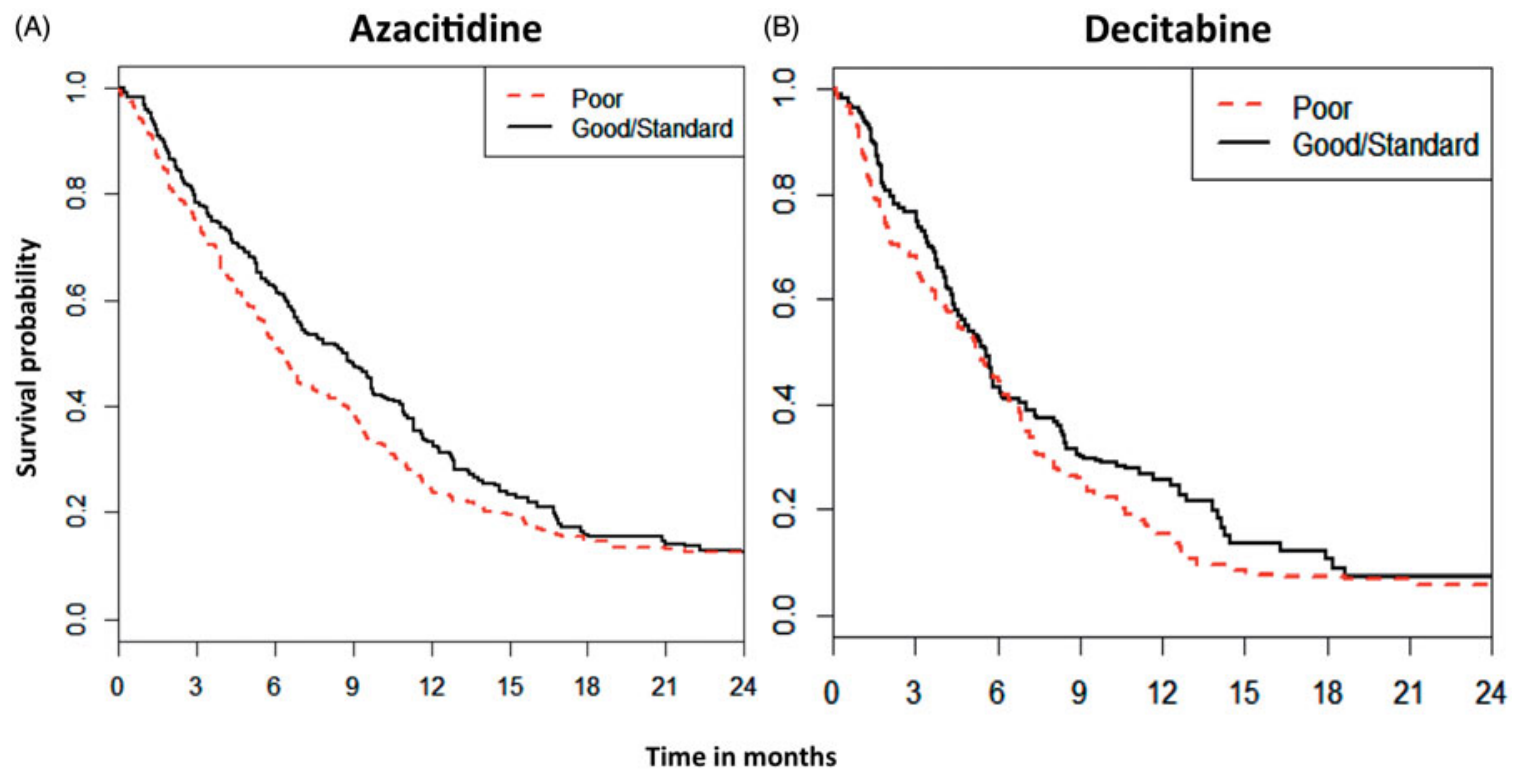

Median OS based on MRC/LRF risk good/standard: 8.5 months poor: 6.2 months

1-year OS probability based on MRC/LRF risk good/standard: $32.7 .2 \%$ (95\% Cl: $24.2-41.4 \%)$ poor: $24.2 \%$ (95\% Cl: $18.1-30.7 \%)$ $\mathrm{p}=0.059$

Median OS based on MRC/LRF risk good/standard: 5.6 months poor: 5.3 months

$p=0.28$

1-year OS probability based on MRC/LRF risk good/standard: $25.7 \%$ (95\% Cl: $15.9-36.7 \%)$ $\mathrm{p}=\mathbf{0 . 3 3}$

poor: $15.4 \%(95 \% \mathrm{Cl}: 9.0-23.3 \%)$

Figure 2: Probability of OS according to MRC/LRF risk category (good/standard vs. poor). (A) From onset of azacitidine treatment; (B) from onset of decitabine treatment. 
included infectious complications (27.7\%), cardiovascular or pulmonary dysfunction (7.5\%); however, only $1.7 \%$ of patients died because of adverse effects directly contributed by the investigators to HMA treatment.

Our study indicates that in contrast to its use in survival prognostication with azacitidine therapy in the frontline setting, the MRC/LRF score has limited ability to discriminate survival with azacitidine or decitabine therapy in the refractory and relapsed setting. This is probably related to the generally dismal survival of these patients and the limited clinical benefit of HMA therapy in this setting. Interestingly, compared with the expanded E-ALMA series population receiving frontline azacitidine therapy, in our population of RR-AML patients the proportion of good risk patients (5.4\% vs. $20.9 \%$ ) was higher, whereas the proportion of poor risk patients $(74.1 \%$ vs. $54.3 \%)$ was lower. However, clearly the patient population in our study was high risk given that patients either demonstrated resistance to prior treatment or relapsed with a short median CR1 time of 8 months for those who achieve a CR1; additionally, $19 \%$ of patients relapsed after HSCT (Table 1).

In a recent analysis of clinical and molecular predictors of response and OS achieved with HMA therapy in RR$A M L$, we found that only patients with low proliferative disease as indicated by peripheral blood blasts $<5 \%$ and bone marrow blast $<20 \%$ at the time of HMA initiation had improved response and OS [3]. Our study emphasizes the extremely poor prognosis of RR-AML patients, who are not eligible for intensive chemotherapy, and argues for aggressive consideration of these patients for enrollment in clinical trials.

Potential conflict of interest: Disclosure forms provided by the authors are available with the full text of this article online at http://dx.doi.org/10.1080/10428194. 2018.1468893.

\section{Funding}

The authors acknowledge the Frederick A. DeLuca Foundation for financial support.

\section{ORCID}

Aref Al-Kali (iD http://orcid.org/0000-0002-0824-3715

Mark Litzow (iD http://orcid.org/0000-0002-9816-6302

Thomas Prebet (D) http://orcid.org/0000-0002-6872-625X

\section{References}

[1] Thol F, Schlenk RF, Heuser M, et al. How I treat refractory and early relapsed acute myeloid leukemia. Blood. 2015;126:319-327.

[2] Al-Ali HK, Jaekel N, Niederwieser D. The role of hypomethylating agents in the treatment of elderly patients with AML. J Geriatr Oncol. 2014;5:89-105.

[3] Stahl M, Devaux M, Montesinos $P$, et al. Hypomethylating agents in relapsed-refractory AML: outcomes and their predictors in a large international patient cohort. Blood Adv. 2018;2:923-932.

[4] Treppendahl MB, Kristensen LS, Gronbaek K. Predicting response to epigenetic therapy. J Clin Invest. 2014;124:47-55.

[5] Falantes J, Pleyer L, Thepot S, et al. Real life experience with frontline azacitidine in a large series of older adults with acute myeloid leukemia stratified by MRC/LRF score: results from the expanded international E-ALMA series (E-ALMA+). Leuk Lymphoma. 2018;5:1113-1120.

[6] van Buuren, S, Groothuis-Oudshoorn, K. Mice: multivariate imputation by chained equations in R. J Stat Soft. 2011;45:1-2. 